\title{
Article \\ Chemical Constituents of the Essential Oil Extracted from Elsholtzia densa and Their Insecticidal Activity against Tribolium castaneum and Lasioderma serricorne
}

\author{
Junyu Liang ${ }^{1,2, *}$, Yazhou Shao ${ }^{1}$, Haoshu Wu ${ }^{1}$, Yue An ${ }^{1}$, Junlong Wang ${ }^{1,2}$, Ji Zhang ${ }^{1,2}$ and Weibao Kong ${ }^{1}$ \\ 1 College of the Life Science, Northwest Normal University, Lanzhou 730070, China; \\ 2018212215@nwnu.edu.cn (Y.S.); lzycsys@163.com (H.W.); 17793510885@163.com (Y.A.); \\ wangjunlong@nwnu.edu.cn (J.W.); zhangj@nwnu.edu.cn (J.Z.); kwbao@163.com (W.K.) \\ 2 New Rural Development Research Institute of Northwest University, Northwest Normal University, \\ Lanzhou 730070, China \\ * Correspondence: $18893811951 @ 163 . c o m$
}

check for updates

Citation: Liang, J.; Shao, Y.; Wu, H.; An, Y.; Wang, J.; Zhang, J.; Kong, W. Chemical Constituents of the Essential Oil Extracted from Elsholtzia densa and Their Insecticidal Activity against Tribolium castaneum and Lasioderma serricorne. Foods 2021, 10, 2304. https://doi.org/10.3390/ foods10102304

Academic Editor: Pascal Degraeve

Received: 19 August 2021

Accepted: 25 September 2021

Published: 28 September 2021

Publisher's Note: MDPI stays neutral with regard to jurisdictional claims in published maps and institutional affiliations.

Copyright: (c) 2021 by the authors Licensee MDPI, Basel, Switzerland. This article is an open access article distributed under the terms and conditions of the Creative Commons Attribution (CC BY) license (https:// creativecommons.org/licenses/by/ $4.0 /)$.

\begin{abstract}
Storage pests pose a great threat to global food security. Here, we found that the essential oil (EO) extracted from E. densa possesses obvious effects against the insects that threaten storedproducts. In this work, we investigated the chemical constituents of the essential oil extracted from Elsholtzia densa, and their insecticidal (contact and fumigant) toxicity against Tribolium castaneum and Lasioderma serricorne. A total of 45 compounds were identified by GC-MS, accounting for $98.74 \%$ of the total EO. Meanwhile, 11 compounds were isolated from the EO, including limonene, $\beta$-caryophyllene, $\rho$-cymene, trans-phytol, $\alpha$-terpineol, linalool, acetophenone, 1,8 -cineole, $\rho$-cymen7-ol, 1-O-cerotoylgly-cerol, and palmitic acid. Furthermore, acetophenone, $\rho$-cymen-7-ol, and 1-Ocerotoylgly-cerol were isolated for the first time from Elsholtzia spp. The results of the bioassays indicated that the EO had the property of insecticidal toxicity against T. castaneum and L. serricorne. All of the compounds showed different levels of insecticidal toxicity against the two species of insects. Among them, 2-ethyl-1H-imidazole had no insecticidal toxicity against $T$. castaneum, but possessed fumigant and obvious contact toxicity against L. serricorne. $\rho$-Cymen-7-ol had beneficial insecticidal toxicity against the two species of insects, and fumigant toxicity against L. serricorne. $\rho$-Cymen-7-ol $\left(\mathrm{LD}_{50}=13.30 \mu \mathrm{g} /\right.$ adult $), 1$-octen-3-ol $\left(\mathrm{LD}_{50}=13.52 \mu \mathrm{g} / \mathrm{adult}\right)$, and 3-octanol $\left(\mathrm{LD}_{50}=17.45 \mu \mathrm{g} / \mathrm{adult}\right)$ showed significant contact toxicity against $T$. castaneum. Acetophenone $\left(\mathrm{LD}_{50}=7.07 \mu \mathrm{g} / \mathrm{adult}\right)$ and $\rho$-cymen-7-ol $\left(\mathrm{LD}_{50}=8.42 \mu \mathrm{g} /\right.$ adult $)$ showed strong contact toxicity against $L$. serricorne. $\rho$-Cymene $\left(\mathrm{LC}_{50}=10.91 \mathrm{mg} / \mathrm{L}\right.$ air$)$ and $\rho$-cymen-7-ol $\left(\mathrm{LC}_{50}=10.47 \mathrm{mg} / \mathrm{L}\right.$ air $)$ showed powerful fumigant toxicity to $T$. castaneum. Limonene $\left(\mathrm{LC}_{50}=5.56 \mathrm{mg} / \mathrm{L}\right.$ air $)$, acetophenone $\left(\mathrm{LC}_{50}=5.47 \mathrm{mg} / \mathrm{L}\right.$ air $)$, and 3-octanol $\left(\mathrm{LC}_{50}=5.05 \mathrm{mg} / \mathrm{L}\right.$ air) showed obvious fumigant toxicity against L. serricorne. In addition, the EO and its chemical compounds possessed different levels of repellent activity. This work provides some evidence of the value of exploring these materials for insecticidal activity, for human health purposes. We suggest that the EO extracted from E. densa may have the potential to be developed as an insecticidal agent against stored product insect pests.
\end{abstract}

Keywords: Elsholtzia densa; insecticidal activity; repellency; Tribolium castaneum; Lasioderma serricorne

\section{Introduction}

As the world's population continues to grow, the demand for food crops is increasing every year. However, a major difficulty in the production and storage of cereal products, grains, and Chinese herbs is insect infestation [1]. Pests are a problem worldwide because of migration, high reproductive rates, and wide distribution. Insect pests play an important role in damaging crops, causing both direct and indirect economic loss [2]. These insect pests are active throughout the year on various crops, and are associated with a loss of more than one billion US dollars per year worldwide [3]. Traditional methods of pest control are 
dependent on chemical synthetic insecticides, because they are highly efficient [4,5]. However, the repeated use of synthetic chemicals to manage pests has resulted in resurgence and outbreaks, resistance to insecticides, the elimination of existing natural enemies, and the pollution of soil, water, air and food [6]. It is necessary to reduce the risks associated with the excessive application of high pesticide doses in agricultural primary production. The growing awareness of the hazards of excessive use of pesticides globally has led researchers to search for safer and more environmentally friendly alternative methods for insect pest control [7]. Hence, the search for viable and sustainable alternatives to synthetic pesticides is vital [8]. Plants have chemical defense mechanisms against insects and other organisms; these defense mechanisms do not generally produce immediate death, but do affect common biochemical and physiological functions [9]. Botanical pesticides tend to have broad-spectrum activity, are relatively specific in their mode of action, and are generally safe to other living organisms and the environment [10]. Therefore, botanical pesticides have been frequently discussed in recent years. Research on natural products that could be alternatives to synthetic pesticides and fungicides, for example plant extracts and essential oils, has therefore greatly increased during recent years [11].

Natural product essential oils (EOs) are extracted from aromatic plants, and consist of terpenes, aromatics, and phenolic components [12]. EOs are generally considered environmentally and medically safe because of their volatility [13]. EOs may significantly reduce the harmfulness of some pests, and thus be worth further attention. There are some reports that EOs possess a wide range of curative potentials, such as antimicrobial, antifungal, antiviral, antioxidant, antifeedant, and insecticidal activities [14]. Among them, the antifeedant and insecticidal activities of many plant extracts, and their bioactive compounds against several insect pests, have been demonstrated. Wang et al. [15] reported that there are obvious toxicity and repellent activities of EOs and their major compounds from four spice plants, against two storage product insects (T. castaneum and L. serricorne). EOs and polyacetylene extracted from Artemisia ordosica possess significant fumigant toxicity and repellent activity against $T$. castaneum, and five polyacetylenes were isolated from Artemisia ordosica [16]. EOs and their compounds extracted from Valerianaceae spp [17], Murraya tetramera Huang [18], Zanthoxylum planispinumvar. Dintanensis [19], and Valeriana officinalis [20] have also been demonstrated to possess potential activity against stored product insects. Plant-derived extracts can also control pests as antifeedant agents.

Elsholtzia bensa belongs to Elsholtzia Willd. It is a widely spread plant in China [21]. $E$. Bensa has antibacterial and antiviral properties that make it excellent for use in the treatment of diseases [22]. However, E. densa is regarded as a weed in many provinces of China. In this work, the chemical compounds of an EO extracted from E. densa were analyzed by GC-MS. We chose T. castaneum and L. serricorne as target insects to evaluate the insecticidal and repellent activity of this EO and its compounds. We expected to find efficacy of $E$. densa, both as an insecticide and repellent, against storage pests.

\section{Materials and Methods}

\subsection{Plant Material and the Extraction of the EO}

The fresh aerial parts of E. densa were collected from Longxi county, Gansu Province, China ( $35^{\circ} 1^{\prime} 23^{\prime \prime}$ N latitude, $104^{\circ} 27^{\prime} 17^{\prime \prime}$ E longitude, altitude $1904.7 \mathrm{~m}$ ) in September 2019. The botanical authentication was performed by Dr. Liang, J. Y. (College of Life Science, Northwest Normal University, Lanzhou, China), and a voucher specimen was deposited in the Herbarium (NWNU) of the College of Life Science, Northwest Normal University, under the number NWNU-20190918002. After the aerial parts of $E$. densa were air-dried at room temperature in the shade, they were cut into small pieces. The EO of $E$. densa were obtained by hydrodistillation for $6 \mathrm{~h}$ on a modified Clevenger-type apparatus, and stored in dark airtight containers in a refrigerator at $4{ }^{\circ} \mathrm{C}$. 


\subsection{Insects}

T. castaneum and L. serricorne were provided by the laboratory of quality research and product development of traditional Chinese medicine, College of Life Science, Northwest Normal University, and were confirmed by Dr. Liang J. Y. (College of Life Science, Northwest Normal University, Lanzhou, China). The two kinds of insects were artificially subcultured and purified for more than 4 to 5 generations in dark incubator at $28-30{ }^{\circ} \mathrm{C}$, with relative humidity maintained at $70-80 \%$. They were reared on a mixture of wheat flour and yeast $(10: 1, w / w)$. The mixed-sex adult insects used in all bioassays were about $1-2$ weeks old.

\subsection{GC-MS Analysis}

This was carried out according to the method reported previously [23]. The chemical compounds of the EO were analyzed by GC-MS. The column of the GC was a quartz capillary column HP-5MS $(30 \mathrm{~m} \times 0.25 \mathrm{~mm} \times 0.25 \mu \mathrm{m})$, the temperature was $250{ }^{\circ} \mathrm{C}$, high purity helium was used as the carrier gas, the flow rate was $1.0 \mathrm{~mL} / \mathrm{min}$, the injection volume was $1.0 \mu \mathrm{L}$, and the split ratio was 100:1. The heating program was set as follows: the initial temperature was $60^{\circ} \mathrm{C}$ and was maintained for $2 \mathrm{~min}$, then the temperature increased to $180{ }^{\circ} \mathrm{C}$ at a rate of $10^{\circ} \mathrm{C} / \mathrm{min}$, was maintained for $1 \mathrm{~min}$, before finally the temperature reached $260^{\circ} \mathrm{C}$ at a rate of $20^{\circ} \mathrm{C} / \mathrm{min}$, prior to holding it for $15 \mathrm{~min}$. EI-MS was carried out at $70 \mathrm{eV}$. The EO samples were diluted in acetone to prepare $1 \%$ solutions. Further identification was made by comparison of their MS with those stored in Wiley 275 and NIST 11, or with the literature [24].

\subsection{Isolation and Identification of Pure Compounds}

The crude EO $(75 \mathrm{~mL})$ of E. densa was chromatographed on a silica gel column (160-200 mesh, Qingdao Marina Chemical Plant, Qingdao, China) (column length $90 \mathrm{~cm}$, diameter $5 \mathrm{~cm}$ ), then eluted with a gradient of petroleum ether-ethyl acetate (from 100:0 to 0:100). Each $100 \mathrm{~mL}$ of eluate was collected as a fraction. With the monitoring of thin layer chromatography (TLC) profiles, similar fractions were combined and at last 10 fractions were obtained. Among them, the fractions (1-5) were pooled and further purified by silica gel column chromatography, and 11 compounds were isolated from them. The isolated compounds were elucidated with nuclear magnetic resonance. ${ }^{1} \mathrm{H}$ and ${ }^{13} \mathrm{C}$ NMR were performed on an NMR spectrometer (Agilent Technologies, $400 \mathrm{MHz}$ (Vnmr mercury400 plus) and $600 \mathrm{MHz}$ (Agilent DD2-600 MHz) for proton) at a temperature of $25^{\circ} \mathrm{C}$ in the deuterated chloroform $\left(\mathrm{CDCl}_{3}\right)$.

\subsection{Bioassay \\ 2.5.1. Contact and Fumigant Activity}

According to the methods of Liu and Ho [25], the drip method and filter paper sheet method were used for the whole process of contact and fumigant activity, respectively. From the raised adults, 10 healthy adults with good activity and consistent growth were selected (regardless of gender). They were placed in an activity test glass bottle $(5.5 \mathrm{~cm}$ high, $2.5 \mathrm{~cm}$ in diameter). The $\mathrm{EO}$ and isolated compounds were dissolved in acetone to prepare a serial testing solution, with acetone solvent as the negative control. According to the results of preliminary experiments, five concentrations of the $\mathrm{EO}$ and its isolated compounds were determined in formal experiments. Each treatment and control of different concentrations was replicated five times. The death/survival of the test insects were observed and recorded $24 \mathrm{~h}$ later, and abnormal activity of the insects was regarded as death.

\subsubsection{Repellant Activity}

Repellant activity was assessed according to the method reported previously [26]. The $\mathrm{EO}$, its isolated compounds, and commercial compounds were dissolved in acetone to prepare a serial testing solution $\left(78.63,15.73,3.15,0.63,0.13 \mathrm{~nL} / \mathrm{cm}^{2}\right)$. Acetone and DEET were used negative and positive controls, respectively. All of the experiments were carried 
out on Petri dishes ( $9 \mathrm{~cm}$ in diameter). A filter paper disk $(9 \mathrm{~cm}$ in diameter) was cut into two halves. One half was uniformly treated with $500 \mu \mathrm{L}$ of a testing solution, and the other half was treated with $500 \mu \mathrm{L}$ of acetone. There were 20 insects in each Petri dish center. The number of insects that stayed on the treated $(N t)$ and control $(N c)$ halves were recorded after 2 and $4 \mathrm{~h}$. Every treatment was repeated five times. Then, the percentage repellency (PR) of the EO and each compound was calculated using the formula:

$$
\operatorname{PR}(\%)=\frac{N c-N t}{N c+N t} \times 100
$$

where $N c$ is the number of insects present in the negative control half, and $N t$ is the number of insects present in the treated half. At the same time, the average repellent rate was graded by reference [27], as shown in the table below. PR values were transferred into arcsin square root values, and subjected to One-Way Analysis of Variance (ANOVA) under Tukey's HSD test at $p<0.05$ (SPSS V 22.0, IBM, New York, NY, USA) (Table 1).

Table 1. Rating sheet of repellency.

\begin{tabular}{ccccccc}
\hline Grade & $\mathbf{0}$ & I & II & III & IV & V \\
\hline PR $(\%)$ & $0.01 \sim 0.1$ & $0.1 \sim 20$ & $20.1 \sim 40$ & $40.1 \sim 60$ & $60.1 \sim 80$ & $80.1 \sim 100$ \\
\hline
\end{tabular}

\subsection{Data Analysis}

In the bioassay of fumigant and contact, the effects of the $\mathrm{EO}$ and compounds were expressed by $\mathrm{LC}_{50}$ and $\mathrm{LD}_{50}$ values. Probit analysis [28] was used for the calculation of these values. $\mathrm{LC}_{50}, \mathrm{LD}_{50}$, and $\mathrm{PR}$ values were transferred into arcsin square root values, and subjected to One-Way Analysis of Variance (ANOVA) under Tukey's HSD test at $p<0.05$ (SPSS V 19.0, IBM, NY, USA).

\subsection{Chemical Compounds}

Eight commercial compounds (furfural, dibutyl phthalate, dioctyl phthalate, geraniol, 1-octen-3-ol, 3-octanol, 2-ethyl-1H-imidazole and 2,4-ditert-butylphenol) were purchased from Saen Chemical Technology Co., Ltd. Shanghai, China, The purity values were 99, 99.5, $98,97,98,98,99$ and $98 \%$, respectively.

\section{Results and Discussion}

\subsection{Chemical Composition of the EO for E. densa}

3.1.1. Yield of EO

The results showed that the yield of EO of E. densa was high at $0.34 \%$, and the relative density of the $\mathrm{EO}$ of $E$. densa was $0.88 \mathrm{~g} / \mathrm{mL}$ (Table 2). In the extraction process of water distillation of the EO, the first extracted oil is a light white light oil. With the extension of the extraction time, a light yellow oil was extracted immediately after the extraction, and was inter-dissolved with the light white translucent light oil. When the extraction time was 5-6 h, the extracted EO was a dark green light oil, and the rate of oil production was obviously reduced, but it was also inter-dissolved with the EO obtained from the previous extraction, and the overall appearance was a dark yellow and opaque oil-like liquid.

Table 2. EO yield of the aerial parts of E. densa.

\begin{tabular}{ccccc}
\hline Name & $\begin{array}{c}\text { Sample Size } \\
\mathbf{( k g})\end{array}$ & Volume $(\mathbf{m L})$ & Yield $(v / w, \%)$ & $\begin{array}{c}\text { Relative } \\
\text { Density }(\mathbf{g} / \mathbf{m L})\end{array}$ \\
\hline E. densa & 24.80 & 84.30 & 0.34 & 0.88 \\
\hline
\end{tabular}

\subsubsection{Chemical Composition of the EO}

The chemical composition of the EO is listed in Table 3. The results show that the EO of E. densa mainly contained terpenes and aromatic compounds, and among the terpene compo- 
nents, mainly monoterpenes and sesquiterpenes were identified. A total of 42 compounds were identified, accounting for $90.25 \%$ of the total EO. Limonene (22.05\%), 2-Methyl-3methylene-1-cyclopentanecarboxylic acid methyl ester (20.00\%), 7-Methyl-3-methylidene-4(propan-2-yl)octahydro-1H-cyclopenta[1,3]cyc-lopropa[1,2]benzene $(11.17 \%)$, and $\rho$-cymen7 -ol $(7.50 \%)$ were the major components. A total of 26 compounds were identified in the EO of E. densa by Liu et al. [29], among which the higher contents were $\alpha$-bisabolol (14.97\%), elemene $(9.04 \%), \beta$-selinene (8.97\%), (+)- $\gamma$-curjunene (6.37\%), 3-methylenecyclopentanol $(6.03 \%), \alpha$-guaiene $(5.92 \%)$, and 1,2,3,4,5,6,7,8-octahydro-1,4-dimethyl-7-(1-methylethyl)tenebrand hydrocarbon (5.92\%). The main components were different from those found in the present study. The reason for the differences maybe that the aerial parts of E. densa were used in our study, and other studies collected samples from different places (the differences may be related to the sampling environment and location) $\rho$-Cymen-7-ol.

Table 3. Chemical composition of essential oil from the E. densa..

\begin{tabular}{|c|c|c|c|c|c|}
\hline $\begin{array}{l}\text { Peak } \\
\text { NO. }\end{array}$ & $\begin{array}{c}\mathrm{RT} \\
(\mathrm{min})\end{array}$ & Compound & $\mathbf{R I}$ * & $\begin{array}{c}\text { Relative } \\
\text { Content }(\%)\end{array}$ & Formula \\
\hline 1 & 3.539 & 3-Thujene & 925 & 0.10 & $\mathrm{C}_{10} \mathrm{H}_{16}$ \\
\hline 2 & 3.653 & 2,6,6-Trimethylbicyclo[3.1.1]hept-2-ene & 939 & 0.41 & $\mathrm{C}_{10} \mathrm{H}_{16}$ \\
\hline 3 & 4.306 & $\beta$-Pinene & 978 & 0.86 & $\mathrm{C}_{10} \mathrm{H}_{16}$ \\
\hline 4 & 4.357 & 1-Octen-3-ol & 986 & 0.67 & $\mathrm{C}_{8} \mathrm{H}_{16} \mathrm{O}$ \\
\hline 5 & 4.414 & 3-Octanol & 994 & 0.16 & $\mathrm{C}_{8} \mathrm{H}_{18} \mathrm{O}$ \\
\hline 6 & 4.683 & $\beta$-Phellandrene & 1028 & 0.39 & $\mathrm{C}_{10} \mathrm{H}_{16}$ \\
\hline 7 & 5.170 & o-Cymene & 1029 & 0.38 & $\mathrm{C}_{10} \mathrm{H}_{14}$ \\
\hline 8 & 5.267 & Limonene & 1035 & 22.05 & $\mathrm{C}_{10} \mathrm{H}_{16}$ \\
\hline 9 & 5.370 & $\beta$-Ocimene & 1037 & 0.33 & $\mathrm{C}_{10} \mathrm{H}_{16}$ \\
\hline 10 & 5.553 & 3,7-Dimethyl-1,3,6-octatriene & 1044 & 5.79 & $\mathrm{C}_{10} \mathrm{H}_{16}$ \\
\hline 11 & 5.719 & $\gamma$-Terpinene & 1061 & 0.81 & $\mathrm{C}_{10} \mathrm{H}_{16}$ \\
\hline 12 & 5.919 & Acetophenone & 1065 & 0.52 & $\mathrm{C}_{8} \mathrm{H}_{8} \mathrm{O}$ \\
\hline 13 & 6.354 & 3-Oxabicyclo[4.3.0]non-8-en-2-one & 1084 & 0.38 & $\mathrm{C}_{9} \mathrm{H}_{12} \mathrm{O}$ \\
\hline 14 & 7.064 & 4-Pyridinol & 1154 & 0.15 & $\mathrm{C}_{5} \mathrm{H}_{5} \mathrm{NO}$ \\
\hline 15 & 7.521 & 2-Methyl-3-methylene-1-cyclopentanecarboxylic acid methyl ester & 1169 & 20.00 & $\mathrm{C}_{9} \mathrm{H}_{14} \mathrm{O}_{2}$ \\
\hline 16 & 7.624 & Bornyl chloride & 1203 & 0.57 & $\mathrm{C}_{10} \mathrm{H}_{17} \mathrm{Cl}$ \\
\hline 17 & 7.848 & 1,3-Dimethylcyclohexene & 1264 & 0.57 & $\mathrm{C}_{8} \mathrm{H}_{14}$ \\
\hline 18 & 8.248 & $\rho$-Cymen-7-ol & 1291 & 7.50 & $\mathrm{C}_{10} \mathrm{H}_{14} \mathrm{O}$ \\
\hline 19 & 9.925 & 1-Methylene-2-methyl-3-isopropenylcyclopentane & 1306 & 0.17 & $\mathrm{C}_{10} \mathrm{H}_{16}$ \\
\hline 20 & 10.457 & $\alpha$-Copaene & 1376 & 0.16 & $\mathrm{C}_{15} \mathrm{H}_{24}$ \\
\hline 21 & 10.588 & $\beta$-Bourbonene & 1384 & 0.56 & $\mathrm{C}_{15} \mathrm{H}_{24}$ \\
\hline 22 & 10.663 & $\beta$-Elemene & 1394 & 0.56 & $\mathrm{C}_{15} \mathrm{H}_{24}$ \\
\hline 23 & 11.058 & $\beta$-Caryophyllene & 1399 & 4.41 & $\mathrm{C}_{15} \mathrm{H}_{24}$ \\
\hline 24 & 11.332 & 1,1,7-Trimethyl-4-methylenedecahydro-Alloaromadendrene & 1433 & 0.10 & $\mathrm{C}_{15} \mathrm{H}_{24}$ \\
\hline 25 & 11.492 & 2,6,6,9-Tetramethyl-1,4,8-cycloundecatriene & 1438 & 3.68 & $\mathrm{C}_{15} \mathrm{H}_{24}$ \\
\hline 26 & 11.607 & 1,2,3,5,6,7,8,8a-Octahydro-1-methyl-6-methylene-4-(1-methylethyl)-naphthalene & 1453 & 0.14 & $\mathrm{C}_{15} \mathrm{H}_{24}$ \\
\hline 27 & 11.773 & $\gamma$-Muurolene & 1474 & 0.18 & $\mathrm{C}_{15} \mathrm{H}_{24}$ \\
\hline 28 & 11.859 & $\begin{array}{l}\text { 7-Methyl-3-methylidene-4-(propan-2-yl)octahydro-1H- } \\
\text { cyclopenta[1,3]cyclopropa[1,2]benzene }\end{array}$ & 1486 & 11.17 & $\mathrm{C}_{15} \mathrm{H}_{24}$ \\
\hline 29 & 11.933 & $\alpha$-Farnesene & 1524 & 1.19 & $\mathrm{C}_{15} \mathrm{H}_{24}$ \\
\hline 30 & 12.030 & Bicyclogermacrene & 1532 & 2.09 & $\mathrm{C}_{15} \mathrm{H}_{24}$ \\
\hline 31 & 12.231 & 1-Isopropyl-7-methyl-4-methylene-1,2,3,4,4a,5,6,8a-octahydronaphthalene & 1558 & 0.27 & $\mathrm{C}_{15} \mathrm{H}_{24}$ \\
\hline 32 & 12.344 & Cadinene & 1572 & 0.76 & $\mathrm{C}_{15} \mathrm{H}_{24}$ \\
\hline 33 & 12.986 & 1-Hydroxy-1,7-dimethyl-4-isopropyl-2,7-cyclodecadiene & 1573 & 0.33 & $\mathrm{C}_{15} \mathrm{H}_{26} \mathrm{O}$ \\
\hline 34 & 13.020 & Spathulenol & 1576 & 0.19 & $\mathrm{C}_{15} \mathrm{H}_{24} \mathrm{O}$ \\
\hline 35 & 13.095 & 1-Ethenyl-1-methyl-2,4-bis(1-methylethenyl)-cyclohexane & 1585 & 0.27 & $\mathrm{C}_{15} \mathrm{H}_{24}$ \\
\hline 36 & 13.398 & Spiro[4.4]nonan-2-one & 1608 & 0.15 & $\mathrm{C}_{9} \mathrm{H}_{14} \mathrm{O}$ \\
\hline 37 & 13.741 & T-Cadinol & 1649 & 0.50 & $\mathrm{C}_{15} \mathrm{H}_{26} \mathrm{O}$ \\
\hline 38 & 13.896 & $\alpha$-Cadinol & 1663 & 0.60 & $\mathrm{C}_{15} \mathrm{H}_{26} \mathrm{O}$ \\
\hline 39 & 14.462 & Decahydro-4a-methyl-1-methylene-7-(1-methylethenyl)-naphthalene & 1731 & 0.28 & $\mathrm{C}_{15} \mathrm{H}_{24}$ \\
\hline 40 & 18.090 & Phytol & 2111 & 0.27 & $\mathrm{C}_{20} \mathrm{H}_{40} \mathrm{O}$ \\
\hline 41 & 19.572 & Bis(2-ethylhexyl) adipate & 2398 & 0.13 & $\mathrm{C}_{22} \mathrm{H}_{42} \mathrm{O}_{4}$ \\
\hline 42 & 23.263 & Didecan-2-yl phthalate & 2956 & 0.45 & $\mathrm{C}_{28} \mathrm{H}_{46} \mathrm{O}_{4}$ \\
\hline Total & & & & 90.25 & \\
\hline
\end{tabular}




\subsection{Structural Analysis of Isolated Compounds}

From the EO of the aerial part of E. densa, 11 compounds (1-11) were isolated and identified. Among them, three compounds (7, 9 and 11) were isolated from the EO of E. densa at first. Terpenoids were isolated as the main components of the EO of E. densa. Terpenoids are generally used as flavors in the food industry, and as fragrances in the cosmetics industry.

Eleven compounds were matched with the corresponding data $\left({ }^{1} \mathrm{H}\right.$ and ${ }^{13} \mathrm{C}$ NMR data) in the literature, and their structures are listed in Figure 1.



Limonene (1)

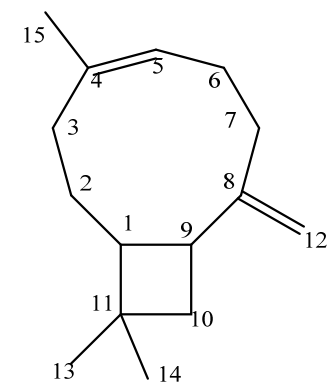

$\beta$-Caryophyllene (2)

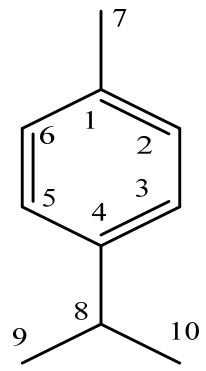

$\rho$-Cymene (3)

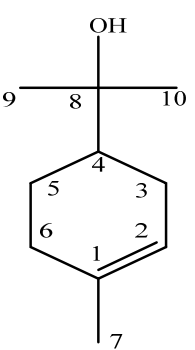

$\alpha$-Terpineol (5)<smiles>CC(=CCCC(C)CCCC(C)=CCO)CCCC(C)P</smiles>

trans-Phytol (4)<smiles>C=CC(C)(O)CCC=C(C)C</smiles>

Linalool (6)

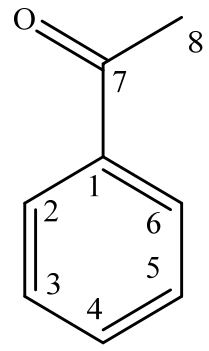

Acetophenone (7)

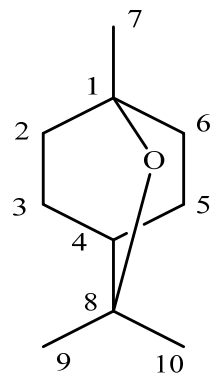

1,8-Cineole (8)



$\rho$-Cymen-7-ol (9)<smiles>CC(C)(CC(C)(C)C(F)(F)F)C(=O)OCC(O)CO</smiles>

1-O-cerotoylgly-cerol (10)

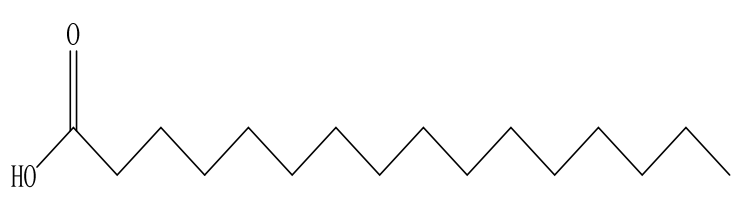

Palmitic acid (11)

Figure 1. Compounds isolated from the essential oil of E. densa.

Limonene (1): colorless oil, $\mathrm{C}_{10} \mathrm{H}_{16} \cdot{ }^{1} \mathrm{H} \mathrm{NMR}\left(\mathrm{CDCl}_{3}, 400 \mathrm{MHz}\right) \delta_{\mathrm{H}}(\mathrm{ppm}): 1.97(2 \mathrm{H}$, $\mathrm{tdd}, J=9.0,3.7,1.8 \mathrm{~Hz}, \mathrm{H}-2), 1.61(2 \mathrm{H}, \mathrm{ddt}, J=11.5,9.8,1.9 \mathrm{~Hz}, \mathrm{H}-3), 1.44(1 \mathrm{H}, \mathrm{dt}, J=8.8$, $1.6 \mathrm{~Hz}, \mathrm{H}-4), 1.18(6 \mathrm{H}, \mathrm{t}, J=1.1 \mathrm{~Hz}, \mathrm{H}-7,10), 1.00(2 \mathrm{H}, \mathrm{d}, J=1.6 \mathrm{~Hz}, \mathrm{H}-5) ;{ }^{13} \mathrm{C} \mathrm{NMR}\left(\mathrm{CDCl}_{3}\right.$, $100 \mathrm{MHz}) \delta_{\mathrm{C}}(\mathrm{ppm}): 150.28$ (C-8), 133.79 (C-1), 120.82 (C-6), 108.48 (C-9), 41.14 (C-4), 30.97 (C-2), 30.75 (C-5), 28.07 (C-3), 23.57 (C-7), 20.91 (C-10). The ${ }^{1} \mathrm{H}$ and ${ }^{13} \mathrm{C}$ NMR data were in agreement with the reported data [30].

$\beta$-Caryophyllene (2): colorless oil, $\mathrm{C}_{15} \mathrm{H}_{24} \cdot{ }^{1} \mathrm{H}$ NMR $\left(\mathrm{CDCl}_{3}, 400 \mathrm{MHz}\right) \delta_{\mathrm{H}}(\mathrm{ppm}): 5.33$ $(1 \mathrm{H}, \mathrm{s}, \mathrm{H}-5), 4.96(1 \mathrm{H}, \mathrm{s}, \mathrm{H}-12 \mathrm{a}), 4.86(1 \mathrm{H}, \mathrm{d}, J=20.0 \mathrm{~Hz}, \mathrm{H}-12 \mathrm{~b}), 2.34(1 \mathrm{H}, \mathrm{q}, J=9.4 \mathrm{~Hz}$, H-9), 2.27-2.17 (4H, m), $2.10(2 \mathrm{H}, \mathrm{d}, J=11.7 \mathrm{~Hz}, \mathrm{H}-1,3 \mathrm{a}), 2.01$ (1H, d, J = 9.3 Hz, H-6a), 1.93 $(1 \mathrm{H}, \mathrm{d}, J=4.9 \mathrm{~Hz}, \mathrm{H}-6 \mathrm{~b}), 1.76-1.40(9 \mathrm{H}, \mathrm{m}), 1.00(3 \mathrm{H}, \mathrm{d}, J=8.9 \mathrm{~Hz}, \mathrm{H}-14) ;{ }^{13} \mathrm{C} \mathrm{NMR}\left(\mathrm{CDCl}_{3}\right.$, $100 \mathrm{MHz}) \delta_{\mathrm{C}}$ (ppm): 16.27 (C-15), 22.63 (C-14), 28.35 (C-13), 29.36 (C-2), 30.06 (C-13), 32.97 
(C-11), 34.79 (C-7), 39.96 (C-3), 40.36 (C-10), 48.47 (C-9), 53.58 (C-1), 111.64 (C-12), 124.31 (C-5), $135.40(\mathrm{C}-4), 154.61(\mathrm{C}-8)$. The ${ }^{1} \mathrm{H}$ and ${ }^{13} \mathrm{C}$ NMR data were in agreement with the reported data [31,32].

$\rho$-Cymene (3): colorless oil, $\mathrm{C}_{10} \mathrm{H}_{14} \cdot{ }^{1} \mathrm{H}$ NMR $\left(\mathrm{CDCl}_{3}, 400 \mathrm{MHz}\right) \delta_{\mathrm{H}}(\mathrm{ppm}): 7.11(4 \mathrm{H}$, s, 2, 3, 5, 6-H), $2.91(1 \mathrm{H}, \mathrm{ddd}, J=12.3,7.7,6.2 \mathrm{~Hz}, \mathrm{H}-8), 2.36(3 \mathrm{H}, \mathrm{d}, J=1.6 \mathrm{~Hz}, \mathrm{H}-7), 1.29$ $(6 \mathrm{H}, \mathrm{dd}, J=7.0,1.5 \mathrm{~Hz}, \mathrm{H}-9,10) ;{ }^{13} \mathrm{C} \mathrm{NMR}\left(\mathrm{CDCl}_{3}, 100 \mathrm{MHz}\right) \delta_{\mathrm{C}}(\mathrm{ppm}): 20.87(\mathrm{C}-7), 24.12$ (C-9, 10), 33.54 (C-8), 126.2 (C-3, 5), 128.93 (C-2, 6), 135.04 (C-1), $145.79(\mathrm{C}-4)$. The ${ }^{1} \mathrm{H}$ and ${ }^{13} \mathrm{C}$ NMR data were in agreement with the reported data [33].

trans-Phytol (4): yellowish oil, $\mathrm{C}_{20} \mathrm{H}_{40} \mathrm{O} .{ }^{1} \mathrm{H} \mathrm{NMR}\left(\mathrm{CDCl}_{3}, 400 \mathrm{MHz}\right) \delta_{\mathrm{H}}(\mathrm{ppm}): 5.39$ $(1 \mathrm{H}, \mathrm{t}, J=7.2 \mathrm{~Hz}, 1-\mathrm{OH}), 4.13(1 \mathrm{H}, \mathrm{dd}, J=7.0,1.6 \mathrm{~Hz}, \mathrm{H}-1), 1.98(1 \mathrm{H}, p, J=8.2 \mathrm{~Hz}, \mathrm{H}-2)$, $1.51(1 \mathrm{H}, \mathrm{dtd}, J=13.2,6.6,1.9 \mathrm{~Hz}, \mathrm{H}-15), 1.46-0.96$ (3H, m, H-20), 0.95-0.74 (12H, m, H-16, $17,18,19) ;{ }^{13} \mathrm{C} \mathrm{NMR}\left(\mathrm{CDCl}_{3}, 100 \mathrm{MHz}\right) \delta_{\mathrm{C}}(\mathrm{ppm}): 140.19$ (C-3), 123.31 (C-2), 59.45 (C-1), 40.00 (C-4), 39.50 (C-14), 37.56 (C-10), 37.49 (C-8), 37.42 (C-12), 36.80 (C-6), 32.91 (C-7), 32.82 (C-11), 28.09 (C-15), 25.27 (C-5), 24.92 (C-13), 24.60 (C-9), 22.83 (C-17), 22.74 (C-16), 19.87 (C-19), 19.83 (C-18), 16.27 (C-20). The ${ }^{1} \mathrm{H}$ and ${ }^{13} \mathrm{C}$ NMR data were in agreement with the reported data [34].

$\alpha$-Terpineol (5): colorless oil, $\mathrm{C}_{10} \mathrm{H}_{18} \mathrm{O} .{ }^{1} \mathrm{H}$ NMR $\left(\mathrm{CDCl}_{3}, 400 \mathrm{MHz}\right) \delta_{\mathrm{H}}(\mathrm{ppm}): 5.34(\mathrm{dh}$, $J=4.0,1.1 \mathrm{~Hz}, 1 \mathrm{H}), 2.10-1.91(2 \mathrm{H}, \mathrm{m}, \mathrm{H}-6), 1.91-1.79(2 \mathrm{H}, \mathrm{m}, \mathrm{H}-3,5), 1.60(4 \mathrm{H}, \mathrm{h}, J=1.2 \mathrm{~Hz}$, $\mathrm{H}-4,7), 1.29-1.18(4 \mathrm{H}, \mathrm{m}, \mathrm{H}-5,10), 1.13(3 \mathrm{H}, \mathrm{d}, J=6.7 \mathrm{~Hz}, \mathrm{H}-9) ;{ }^{13} \mathrm{C} \mathrm{NMR}\left(\mathrm{CDCl}_{3}, 100 \mathrm{MHz}\right)$ $\delta_{\mathrm{C}}(\mathrm{ppm}): 133.96(\mathrm{C}-1), 120.65$ (C-6), 72.72 (C-7), 44.91 (C-4), 30.93 (C-2), 27.44 (C-8), 27.05 (C-3), 26.26 (C-9), 24.02 (C-5), 23.4 (C-10). The ${ }^{1} \mathrm{H}$ and ${ }^{13} \mathrm{C}$ NMR data were in agreement with the reported data $[33,35]$.

Linalool (6): colorless oil, $\mathrm{C}_{10} \mathrm{H}_{18} \mathrm{O} .{ }^{1} \mathrm{H}$ NMR $\left(\mathrm{CDCl}_{3}, 400 \mathrm{MHz}\right) \delta_{\mathrm{H}}(\mathrm{ppm}): 5.85(1 \mathrm{H}$, $\mathrm{dd}, J=17.3,10.8 \mathrm{~Hz}, \mathrm{H}-2), 5.17(1 \mathrm{H}, \mathrm{d}, J=1.4 \mathrm{~Hz}, \mathrm{H}-1$, trans), $5.13(1 \mathrm{H}, \mathrm{d}, J=1.4 \mathrm{~Hz}, \mathrm{H}-6)$, $5.06(1 \mathrm{H}, \mathrm{ddt}, J=8.6,7.2,1.4 \mathrm{~Hz}, \mathrm{H}-1), 5.00(2 \mathrm{H}, \mathrm{d}, J=1.4 \mathrm{~Hz}, \mathrm{H}-5), 2.07-1.88(1 \mathrm{H}, \mathrm{m}, 3-\mathrm{OH})$, 1.66-1.59 (6H, m, H-8, 9), $1.54(2 \mathrm{H}, \mathrm{d}, J=1.3 \mathrm{~Hz}, \mathrm{H}-4), 1.22(3 \mathrm{H}, \mathrm{s}, \mathrm{H}-10) ;{ }^{13} \mathrm{C} \mathrm{NMR}\left(\mathrm{CDCl}_{3}\right.$, $100 \mathrm{MHz}) \delta_{\mathrm{C}}$ (ppm): 144.84 (C-2), 131.65 (C-7), 124.64 (C-6), 111.63 (C-1), 73.37 (C-3), 42.13 (C-5), 28.26 (C-8), 25.65 (C-9), $22.67\left(3-\mathrm{CH}_{3}\right), 17.62(\mathrm{C}-4)$. The ${ }^{1} \mathrm{H}$ and ${ }^{13} \mathrm{C}$ NMR data were in agreement with the reported data $[36,37]$.

Acetophenone (7): yellowish oil, $\mathrm{C}_{8} \mathrm{H}_{8} \mathrm{O} .{ }^{1} \mathrm{H} \mathrm{NMR}\left(\mathrm{CDCl}_{3}, 400 \mathrm{MHz}\right) \delta_{\mathrm{H}}(\mathrm{ppm})$ : 7.91-7.80 (2H, m, H-3, 5), 7.51-7.39 (1H, m, H-4), 7.40-7.24 (2H, m, H-2, 6), 2.46 (3H, d, $J=1.0 \mathrm{~Hz}, \mathrm{H}-8) ;{ }^{13} \mathrm{C} \mathrm{NMR}\left(\mathrm{CDCl}_{3}, 100 \mathrm{MHz}\right) \delta_{\mathrm{C}}(\mathrm{ppm}): 197.69$ (C-7), $136.88(\mathrm{C}-2), 132.83$ (C-3), 128.58 (C-5), 127.74 (C-6), 26.27 (C-8). The ${ }^{1} \mathrm{H}$ and ${ }^{13} \mathrm{C}$ NMR data were in agreement with the reported data [38].

1, 8-Cineole (8): colorless oil, $\mathrm{C}_{10} \mathrm{H}_{18} \mathrm{O} .{ }^{1} \mathrm{H} \mathrm{NMR}\left(\mathrm{CDCl}_{3}, 400 \mathrm{MHz}\right) \delta_{\mathrm{H}}(\mathrm{ppm})$ : 2.00-1.90 (2H, m, H-2), 1.63-1.54 (6H, m, H-3, 6), 1.43 (1H, dt, J = 8.6, $1.6 \mathrm{~Hz}, \mathrm{H}-4), 1.17(6 \mathrm{H}$, $\mathrm{d}, J=1.0 \mathrm{~Hz}, \mathrm{H}-9,10), 0.98(3 \mathrm{H}, \mathrm{d}, J=1.0 \mathrm{~Hz}, \mathrm{H}-7) ;{ }^{13} \mathrm{C} \mathrm{NMR}\left(\mathrm{CDCl}_{3}, 100 \mathrm{MHz}\right) \delta_{\mathrm{C}}(\mathrm{ppm})$ : 73.52 (C-8), 69.70 (C-1), 32.86 (C-4), 31.39 (C-3, 5), 28.85 (C-2, 6), 27.47 (C-7), 22.75 (C-9, 10). The ${ }^{1} \mathrm{H}$ and ${ }^{13} \mathrm{C}$ NMR data were in agreement with the reported data [39].

$\rho$-Cymen-7-ol (9): colorless oil, $\mathrm{C}_{10} \mathrm{H}_{14} \mathrm{O} .{ }^{1} \mathrm{H}$ NMR $\left(\mathrm{CDCl}_{3}, 400 \mathrm{MHz}\right) \delta_{\mathrm{H}}(\mathrm{ppm}): 4.60$ (2H, s, C-7), $2.99(1 \mathrm{H}, p, J=6.9 \mathrm{~Hz}, \mathrm{C}-8), 1.46-1.20(6 \mathrm{H}, \mathrm{m}, \mathrm{C}-9,10) ;{ }^{13} \mathrm{C} \mathrm{NMR}\left(\mathrm{CDCl}_{3}\right.$, $100 \mathrm{MHz}) \delta_{\mathrm{C}}(\mathrm{ppm}): 148.08$ (C-1), 138.38 (C-4), 127.05 (C-2, 3), $126.31(\mathrm{C}-5,6), 64.47(\mathrm{C}-7)$, $33.66(\mathrm{C}-8), 24.00(\mathrm{C}-9,10)$. The ${ }^{1} \mathrm{H}$ and ${ }^{13} \mathrm{C}$ NMR data were in agreement with the reported data [40].

1-O-cerotoylgly-cerol (10): white powder, $\mathrm{C}_{29} \mathrm{H}_{58} \mathrm{O}_{4} \cdot{ }^{1} \mathrm{H} \mathrm{NMR}\left(\mathrm{CDCl}_{3}, 400 \mathrm{MHz}\right) \delta_{\mathrm{H}}$ (ppm): $4.28(1 \mathrm{H}, \mathrm{dd}, J=12.0,4.3 \mathrm{~Hz}), 4.14(1 \mathrm{H}, \mathrm{dd}, J=11.9,6.0 \mathrm{~Hz}), 2.30(3 \mathrm{H}, \mathrm{td}, J=7.6$, $3.6 \mathrm{~Hz}), 1.60(3 \mathrm{H}, \mathrm{ddt}, J=10.6,7.4,3.1 \mathrm{~Hz}), 1.55(3 \mathrm{H}, \mathrm{s}), 1.25(27 \mathrm{H}, \mathrm{s}), 0.87(3 \mathrm{H}, \mathrm{t}, J=7.0 \mathrm{~Hz}$, $\left.26^{\prime}-\mathrm{Me}\right) ;{ }^{13} \mathrm{C} \mathrm{NMR}\left(\mathrm{CDCl}_{3}, 100 \mathrm{MHz}\right) \delta_{\mathrm{C}}(\mathrm{ppm}): 69.13(\mathrm{C}-2), 65.28(\mathrm{C}-1), 62.26(\mathrm{C}-3), 34.22$ $\left(\mathrm{C}-2^{\prime}\right), 32.08\left(\mathrm{C}-24^{\prime}\right), 29.86,29.43,29.28,29.24\left(\mathrm{C}-4^{\prime} \sim 23^{\prime}\right), 25.02\left(\mathrm{C}-3^{\prime}\right), 22.85\left(\mathrm{C}-25^{\prime}\right), 14.27$ $\left(\mathrm{C}-26^{\prime}\right)$. The ${ }^{1} \mathrm{H}$ and ${ }^{13} \mathrm{C}$ NMR data were in agreement with the reported data [41].

Palmitic acid (11): white powder, $\mathrm{C}_{16} \mathrm{H}_{32} \mathrm{O}_{2} \cdot{ }^{1} \mathrm{H} \mathrm{NMR}\left(\mathrm{CDCl}_{3}, 400 \mathrm{MHz}\right) \delta_{\mathrm{H}}(\mathrm{ppm})$ : $2.31(\mathrm{td}, \mathrm{J}=7.6,3.6 \mathrm{~Hz}, 2 \mathrm{H}), 1.61(\mathrm{td}, \mathrm{J}=7.1,4.1 \mathrm{~Hz}, 2 \mathrm{H}), 1.25(\mathrm{~s}, 24 \mathrm{H}), 0.88(\mathrm{t}, \mathrm{J}=7.0 \mathrm{~Hz}, 3 \mathrm{H})$;

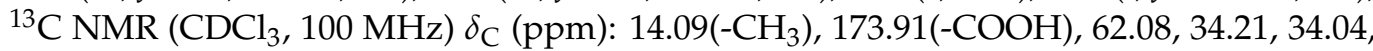


$31.90,29.68,29.64,29.61,29.34,29.25,29.10,29.06,24.85,22.67\left(-\mathrm{CH}_{2}\right)$. The ${ }^{1} \mathrm{H}$ and ${ }^{13} \mathrm{C}$ NMR data were in agreement with the reported data [42].

\subsection{Bioassay}

\subsubsection{Contact Activity}

The contact activities of E. densa against T. castaneum and L. serricorne is listed in Table 4. The EO of E. densa showed obvious contact activity against T. castaneum and L. serricorne, with $\mathrm{LD}_{50}$ values of 29.20 and $24.29 \mu \mathrm{g} /$ adult, respectively. When compared with positive control pyrethrins, the EO demonstrated weak contact activity against T. castaneum and L. serricorne. However, compared with other EOs described in the literature, with which similar bioassays were used, the EO of E. densa possessed stronger contact activity against T. castaneum and L. serricorne. This included in comparison to the EOs of Ajania fruticulosa, Evodia lepta and Citrus wilsonii, which exhibited contact activity against T.castaneum at $\mathrm{LD}_{50}$ values of $105.67,166.94$ and $48.49 \mu \mathrm{g} /$ adult, respectively [43-45]. Meanwhile, the EO of Zingiber zerumbet showed contact activity against L. serricorne, with an $\mathrm{LD}_{50}$ value of $48.3 \mu \mathrm{g} /$ adult [46].

Table 4. Contact activity of compounds against T. castaneum and L. serricorne.

\begin{tabular}{|c|c|c|c|c|c|c|}
\hline Target & Compounds & $\begin{array}{c}\mathrm{LD}_{50} \\
\text { ( } \mu \mathrm{g} / \text { Adult) }\end{array}$ & $95 \%$ FL & Slope \pm SE & $\begin{array}{l}\text { Chi Square } \\
\qquad\left(\chi^{2}\right)\end{array}$ & $p$-Value \\
\hline \multirow{19}{*}{ T. castaneum } & $\mathrm{EO}$ & 29.70 & $14.00-40.40$ & $0.12 \pm 0.03$ & 1.02 & 0.99 \\
\hline & Limonene & 46.61 & $39.19-53.38$ & $0.12 \pm 0.02$ & 6.92 & 1.00 \\
\hline & $\beta$-Caryophyllene & 80.70 & $67.93-96.59$ & $0.07 \pm 0.01$ & 10.14 & 0.93 \\
\hline & $\rho$-Cymene & 33.84 & $31.00-36.64$ & $0.35 \pm 0.05$ & 1.71 & 1.00 \\
\hline & Phytol & 124.26 & $112.01-136.55$ & $0.07 \pm 0.01$ & 6.25 & 1.00 \\
\hline & $\alpha$-Terpineol & 86.17 & 76.38-96.69 & $0.09 \pm 0.01$ & 7.89 & 0.99 \\
\hline & Linalool & 69.12 & $63.67-75.15$ & $0.16 \pm 0.02$ & 4.45 & 1.00 \\
\hline & Acetophenone & 55.80 & $49.04-63.47$ & $0.12 \pm 0.02$ & 23.14 & 4.45 \\
\hline & 1,8-Cineole & 64.21 & $59.11-67.94$ & $0.19 \pm 0.02$ & 5.31 & 1.00 \\
\hline & $\rho$-Cymen-7-ol & 13.30 & $11.44-15.15$ & $0.05 \pm 0.07$ & 5.22 & 1.00 \\
\hline & Furfural & 58.58 & $51.47-65.44$ & $0.12 \pm 0.01$ & 5.84 & 1.00 \\
\hline & Dibutyl phthalate & 105.10 & 94.50-119.42 & $0.08 \pm 0.01$ & 7.44 & 0.99 \\
\hline & Dioctyl phthalate & 116.90 & 104.82-135.09 & $0.08 \pm 0.01$ & 12.23 & 0.97 \\
\hline & Geraniol & 64.08 & $54.11-75.52$ & $0.08 \pm 0.01$ & 11.73 & 0.98 \\
\hline & 1-Octen-3-ol & 13.52 & $10.25-16.54$ & $0.30 \pm 0.04$ & 11.73 & 0.97 \\
\hline & 3-Octanol & 17.45 & 14.19-20.42 & $0.26 \pm 0.03$ & 7.99 & 0.99 \\
\hline & 2-Ethyl-1H-imidazole & $>300$ & & & & \\
\hline & 2,4-Ditert-butylphenol & 53.49 & $45.67-62.34$ & $0.09 \pm 0.01$ & 4.72 & 1.00 \\
\hline & Pyrethrins & 0.10 & $0.08-0.13$ & $2.40 \pm 0.39$ & 8.23 & 0.79 \\
\hline \multirow{19}{*}{ L. serricorne } & $\mathrm{EO}$ & 24.29 & 20.94-27.61 & $0.55 \pm 0.11$ & 1.06 & 0.99 \\
\hline & Limonene & 59.78 & $55.02-64.97$ & $5.43 \pm 0.57$ & 11.26 & 0.98 \\
\hline & $\beta$-Caryophyllene & 45.86 & $42.59-49.20$ & $7.30 \pm 0.85$ & 4.25 & 1.00 \\
\hline & $\rho$-Cymene & 47.56 & $44.18-51.04$ & $7.18 \pm 0.82$ & 5.72 & 1.00 \\
\hline & Phytol & 46.88 & $42.54-51.97$ & $4.56 \pm 0.51$ & 7.25 & 0.99 \\
\hline & $\alpha$-Terpineol & 11.64 & $10.15-13.22$ & $4.01 \pm 0.44$ & 5.34 & 1.00 \\
\hline & Linalool & 16.69 & $14.75-18.97$ & $3.30 \pm 0.32$ & 18.90 & 0.90 \\
\hline & Acetophenone & 7.07 & $6.72-7.43$ & $10.34 \pm 1.21$ & 5.10 & 1.00 \\
\hline & 1,8-Cineole & 11.01 & $9.88-12.25$ & $4.22 \pm 0.45$ & 9.93 & 0.99 \\
\hline & $\rho$-Cymen-7-ol & 8.42 & $6.98-9.81$ & $3.34 \pm 0.42$ & 6.36 & 1.00 \\
\hline & Furfural & 10.21 & $8.08-12.47$ & $3.19 \pm 0.52$ & 2.24 & 1.00 \\
\hline & Dibutyl phthalate & 30.87 & $23.69-41.78$ & $2.06 \pm 0.30$ & 6.68 & 0.92 \\
\hline & Dioctyl phthalate & 24.04 & 18.88-31.05 & $2.36 \pm 0.33$ & 4.07 & 0.99 \\
\hline & Geraniol & 3.14 & $2.29-4.14$ & $2.12 \pm 0.30$ & 5.68 & 0.96 \\
\hline & 1-Octen-3-ol & 3.39 & $2.57-4.38$ & $2.42 \pm 0.33$ & 5.37 & 0.94 \\
\hline & 3-Octanol & 7.75 & $6.45-9.30$ & $4.29 \pm 0.62$ & 3.08 & 0.99 \\
\hline & 2-Ethyl-1H-imidazole & 31.38 & $25.05-40.17$ & $2.64 \pm 0.36$ & 3.77 & 0.99 \\
\hline & 2,4-Di-tert-butylphenol & 32.19 & $26.26-40.26$ & $4.23 \pm 0.35$ & 3.16 & 0.99 \\
\hline & Pyrethrins & 0.09 & $0.07-0.11$ & $3.07 \pm 0.36$ & 6.19 & 0.99 \\
\hline
\end{tabular}


For T. castaneum, only 2-ethylimidazole possessed no contact activity ( $\mathrm{LD}_{50}>300 \mu \mathrm{g} / \mathrm{adult}$ ), even at the highest testing concentration $(50 \%, v / v)$, while the other 16 compounds showed different levels of contact activity. Among them, $\rho$-cymen-7-ol, 1-octen-3-ol and 3-octanol showed significant contact activity against $T$. castaneum, with $\mathrm{LD}_{50}$ values of $13.30,13.52$ and $17.45 \mu \mathrm{g} /$ adult, respectively. Phytol, disobutyl phthalate, and dioctyl phthalate exhibited weak contact activity, with $\mathrm{LD}_{50}$ values of more than $100 \mu \mathrm{g} /$ adult. For L. serricorne, 17 testing compounds exhibited strong contact activity at all the assayed concentrations. Most notably, $\rho$-cymen-7-ol, acetophenone, geraniol, 1-octen-3-ol, and 3-octanol showed very significant contact activities against $L$. serricorne, with $\mathrm{LD}_{50}$ values of 8.42, 7.07, 3.14, 3.39 and $7.75 \mu \mathrm{g} /$ adult, respectively.

Based on the above experimental results, we found that the contact activity of the EO was significantly weaker than that of the some compounds against T. castaneum and L. serricorne, suggesting that complex mixtures of active ingredients might also be beneficial in terms of pest resistance and behavioral desensitization. The different compounds showed different levels of contact activity against T. castaneum and L. serricorne, which may be due to the different sensitivity of different insects to the substances. As the temperature rises [47], the contact activity may become less effective.

\subsubsection{Fumigant Activity}

The fumigant activity of E. densa $\mathrm{EO}$ and its components against T. castaneum and L. serricorne is described in Table 5. The EO of E. densa showed significant fumigant activity against $T$. castaneum and $L$. serricorne, with $\mathrm{LC}_{50}$ values of 18.45 and $14.49 \mathrm{mg} / \mathrm{L}$ air, respectively. The results showed that T. castaneum was more tolerant than L. serricorne. When compared with the famous botanical insecticide, $\mathrm{MeBr}$ and Phosphine, the EO demonstrated weak fumigant activity against T. castaneum and L. serriocorne. However, compared with other EOs tested using a similar bioassay in the literature, the EO of $E$. densa in the present study exhibited stronger fumigant activity against $T$. castaneum and L. serricorne, e.g., EOs of Amomum maximum and Litsea cubeba exhibited fumigant activity against $T$. castaneum at $\mathrm{LC}_{50}$ values of 23.09 and $22.97 \mathrm{mg} / \mathrm{L}$ air, respectively $[48,49]$. The EOs of Alpinia kwangsiensis and Amomum tsaoko showed fumigant activity against L. serricorne at $\mathrm{LC}_{50}$ values of 9.91 and $8.70 \mathrm{mg} / \mathrm{L}$ air, respectively [50,51]. The results demonstrated that T. castaneum is more tolerant than L. serricorne.

In the fumigant activity testing against $T$. castaneum, nine compounds ( $\beta$-caryophyllene, phytol, $\alpha$-terpineol, 1,8-cineole, dibutyl phthalate, dioctyl phthalate, geraniol, 2 -ethyl-1Himidazole, and 2,4-ditert-butylphenol) did not show fumigant activity against T. castaneum at the highest tested concentration. Among the 17 compounds tested, all the tested compounds exhibited obvious activity against T. astaneun, except for nine compounds, including $\beta$-caryophyllene, which did not exhibit fumigant activity against T. castaneum at the highest tested concentration $(50 \%) . \rho$-cymene $\left(\mathrm{LC}_{50}=10.91 \mathrm{mg} / \mathrm{L}\right.$ air $)$ and $\rho$-cymen-7-ol $\left(\mathrm{LC}_{50}=10.47 \mathrm{mg} / \mathrm{L}\right.$ air$)$ exhibited stronger and closer fumigant toxicity against $T$. castaneum . In addition, 1-octen-3-ol and 3-octanol also exhibited relatively similar fumigant toxicity against $T$. castaneum, with $\mathrm{LC}_{50}$ values of 19.17 and $19.85 \mathrm{mg} / \mathrm{L}$ air, respectively.

In the fumigant activity test experiment against L. serricorne, five compounds (phytol, $\rho$-cymen-7-ol, dibutyl phthalate, dioctyl phthalate, and geraniol) did not lead to death of the tested L. serricorne at the highest tested concentration (50\%); they did not exhibit fumigant toxicity against $L$. serricorne. Other compounds exhibited obvious fumigant toxicity against $L$. serricorne. Among them, limonene $\left(\mathrm{LC}_{50}=5.86 \mathrm{mg} / \mathrm{L}\right.$ air), acetophenone $\left(\mathrm{LC}_{50}=5.47 \mathrm{mg} / \mathrm{L}\right.$ air$)$, and 3-octanol $\left(\mathrm{LC}_{50}=5.05 \mathrm{mg} / \mathrm{L}\right.$ air $)$ showed stronger fumigant toxicity against $L$. serricorne, with $\mathrm{LC}_{50}$ values of less than $6.00 \mathrm{mg} / \mathrm{L}$ air. In general, the fumigant activity of $\rho$-cymen-7-ol and $\alpha$-terpineol exhibited significant fumigant activity against L. serricorne, but no fumigant activity against L. serricorne at the highest tested concentration (50\%) was found with $\rho$-cymen-7-ol. Only limonene, $\rho$-cymene, linalool, acetophenone, furfural, 1-octen-3-ol, and 3-octanol showed more stable fumigant activity against both target insects. In contrast, phytol, dibutyl phthalate, dioctyl phthalate, and 
geraniol did not show fumigant toxicity to both target insects. As previously found, temperature can significantly affect the efficiency of EOs. In the fumigant activity test experiment, as the temperature rises, the results may be better.

Table 5. Fumigant activity of compounds against T. castaneum and L. serricorne.

\begin{tabular}{|c|c|c|c|c|c|c|}
\hline Target & Compounds & $\mathrm{LC}_{50}$ (mg/L Air) & $95 \% \mathrm{FL}$ & Slope \pm SE & Chi Square $\left(\chi^{2}\right)$ & $p$-Value \\
\hline \multirow{19}{*}{ T. castaneum } & $\mathrm{EO}$ & 18.45 & $14.89-21.62$ & $0.42 \pm 0.10$ & 0.82 & 0.99 \\
\hline & Limonene & 25.94 & $23.35-28.31$ & $0.32 \pm 0.05$ & 2.08 & 1 \\
\hline & $\beta$-Caryophyllene & $>200$ & & & & \\
\hline & $\rho$-Cymene & 10.91 & $9.45-12.39$ & $0.56 \pm 0.07$ & 4.12 & 1 \\
\hline & Phytol & $>200$ & & & & \\
\hline & $\alpha$-Terpineol & $>200$ & & & & \\
\hline & Linalool & 16.46 & $14.18-18.48$ & $0.37 \pm 0.05$ & 5.05 & 1 \\
\hline & Acetophenone & 37.55 & $28.39-49.52$ & $0.16 \pm 0.02$ & 83.45 & 0 \\
\hline & 1,8-Cineole & $>200$ & & & & \\
\hline & $\rho$-Cymen-7-ol & 10.47 & $8.92-11.99$ & $0.53 \pm 0.07$ & 3.82 & 1 \\
\hline & Furfural & 23.16 & $21.26-25.13$ & $0.40 \pm 0.05$ & 10.38 & 0.99 \\
\hline & Dibutyl phthalate & $>200$ & & & & \\
\hline & Dioctyl phthalate & $>200$ & & & & \\
\hline & Geraniol & $>200$ & & & & \\
\hline & 1-Octen-3-ol & 19.17 & $16.77-21.38$ & $0.03 \pm 0.04$ & 7.61 & 0.99 \\
\hline & 3-Octanol & 19.85 & $17.09-22.34$ & $0.27 \pm 0.03$ & 2.47 & 1 \\
\hline & 2-Ethyl-1H-imidazole & $>200$ & & & & \\
\hline & 2,4-Ditert-butylphenol & $>200$ & & & & \\
\hline & $\mathrm{MeBr}$ & 0.18 & $0.17-0.20$ & $5.71 \pm 0.21$ & 5.63 & 0.96 \\
\hline \multirow{19}{*}{ L. serricorne } & $\mathrm{EO}$ & 14.49 & $12.44-17.12$ & $0.61 \pm 0.12$ & 3.34 & 0.97 \\
\hline & Limonene & 5.86 & $18.34-23.47$ & $4.03 \pm 0.46$ & 9.22 & 0.99 \\
\hline & $\beta$-Caryophyllene & 153.09 & $101.82-514.78$ & $1.79 \pm 0.51$ & 1.95 & 1 \\
\hline & $\rho$-Cymene & 29.06 & $27.03-31.22$ & $7.26 \pm 0.85$ & 6.45 & 1 \\
\hline & Phytol & $>200$ & & & & \\
\hline & $\alpha$-Terpineol & 6.90 & $5.69-8.07$ & $3.18 \pm 0.38$ & 8.19 & 0.99 \\
\hline & Linalool & 10.85 & $9.68-12.08$ & $5.47 \pm 0.63$ & 5.36 & 0.99 \\
\hline & Acetophenone & 5.47 & $5.20-5.92$ & $10.33 \pm 1.26$ & 5.56 & 1 \\
\hline & 1,8-Cineole & 9.57 & $7.99-11.20$ & $2.820 \pm 0.32$ & 3.86 & 1 \\
\hline & $\rho$-Cymen-7-ol & $>200$ & & & & \\
\hline & Furfural & 7.02 & $5.60-8.75$ & $2.64 \pm 0.36$ & 3.94 & 0.99 \\
\hline & Dibutyl phthalate & $>200$ & & & & \\
\hline & Dioctyl phthalate & $>200$ & & & & \\
\hline & Geraniol & $>200$ & & & & \\
\hline & 1-Octen-3-ol & 6.71 & $5.61-8.04$ & $3.81 \pm 0.53$ & 7.29 & 0.88 \\
\hline & 3-Octanol & 5.05 & $4.38-6.61$ & $3.09 \pm 0.44$ & 6.11 & 0.94 \\
\hline & 2-Ethyl-1H-imidazole & 95.85 & $80.12-131.75$ & $3.55 \pm 0.73$ & 2.77 & 0.99 \\
\hline & 2,4-Ditert-butylphenol & 50.41 & $37.82-68.15$ & $1.84 \pm 0.42$ & 2.12 & 1 \\
\hline & Phosphine* & $9.23 \times 10^{-3}$ & $(7.13 \sim 11.37) \times 10^{-3}$ & $2.12 \pm 0.27$ & 11.96 & \\
\hline
\end{tabular}

* as positive control, data from [52].

\subsubsection{Repellent Activity}

The results of repellency assays for the $\mathrm{EO}$ and individual compounds selected against T. castaneum and L. serricorne are shown in Figure 2. The results showed that at the testing concentrations, the $\mathrm{EO}$ and compounds exhibited different levels of repellent activity against $T$. castaneum and $L$. serricorne. Total repellent activity against $T$. castaneum was greater than that for L. serricorne. Acetophenone, $\rho$-cymen-7-ol, $\alpha$-terpineol, and the EO showed strong repellent activities against $T$. castaneum at all testing concentrations, according to most data distributions in Figure 2. It was noteworthy that only linalool possessed weak repellent activity against $T$. castaneum, while the $\mathrm{EO}$ and seven compounds showed significant repellent activity at 78.63 and $15.73 \mathrm{~nL} / \mathrm{cm}^{2}$ at $2 \mathrm{~h}$ after exposure, and had PR values of almost $100 \%$. At the testing concentration range of $3.15-0.13 \mathrm{~nL} / \mathrm{cm}^{2}, \mathrm{EO}$, limonene, $\alpha$-terpineol, acetophenone, and $\rho$-cymen-7-ol possessed significant repellency against T. castaneum, with PR values of $80-90 \%$ at $2 \mathrm{~h}$ post-exposure. $\beta$-Caryophyllene, $\rho$-cymene, phytol, and linalool exhibited mild repellent activity with the testing concentration range of $3.15-0.13 \mathrm{~nL} / \mathrm{cm}^{2}$ at $2 \mathrm{~h}$ after exposure. 1,8-Cineole displayed significant repellent activity at all testing concentrations, with PR values of 100, 94, 74, 64 and $48 \%$, 
respectively, at $2 \mathrm{~h}$ after exposure, and the activity increased with the concentration of exposure. Although seven compounds showed repellent activity against T. castaneum that was greater than that of EO, phytol, and linalool at the highest testing concentration at $4 \mathrm{~h}$ after exposure, these substances had PR values of 98,88 and $86 \%$. The PR values of $\beta$-caryophyllene, acetophenone, and $\rho$-cymen-7-ol showed significant repellent activity of almost $100 \%$ at a testing concentration of $15.73 \mathrm{~nL} / \mathrm{cm}^{2}$ against $T$. castaneum at $4 \mathrm{~h}$ after exposure, while the PR values of $\beta$-caryophyllene, acetophenone, and $\rho$-cymen-7-ol were the same as the positive control DEET at $15.73 \mathrm{~nL} / \mathrm{cm}^{2}$ against $T$. castaneum at $4 \mathrm{~h}$ after exposure. The results indicated the EO and compounds possessed approximately equal repellent activity with DEET against $T$. castaneum at the testing concentrations of 78.63 and $15.73 \mathrm{~nL} / \mathrm{cm}^{2}$ at 2 and $4 \mathrm{~h}$ post-exposure. Unfortunately, $\rho$-cymene showed weak repellent activity against $T$. castaneum with the testing concentration range of $3.15-0.13 \mathrm{~nL} / \mathrm{cm}^{2}$ at $4 \mathrm{~h}$ after exposure, while other compounds exhibited strong repellent activity; it had PR values of $40-80 \%$.

For L. serricorne, $\beta$-caryophyllene, 1,8-cineole and $\rho$-cymene exhibited significant repellent activity when applied at all testing concentrations, and had PR values ranging from $50-100 \%$, at the same level as DEET. The EO, acetophenone and $\rho$-cymen-7-ol showed obvious repellency only at the testing concentrations of 78.63 and $15.73 \mathrm{~nL} / \mathrm{cm}^{2}$. At $4 \mathrm{~h}$ after exposure, $\beta$-caryophyllene, $\rho$-cymene, and $\rho$-cymen-7-ol were better than the EO and other compounds at 78.63 and $15.73 \mathrm{~nL} / \mathrm{cm}^{2}$, according to PR values. $\alpha$-Terpineol and phytol exhibited moderate repellency against L. serricorne at $4 \mathrm{~h}$ after exposure. Limonene and linalool exhibited weak repellent activity against $L$. serricorne at all testing concentrations at $4 \mathrm{~h}$ after exposure. Most notably, at the lowest testing concentration, $\rho$-cymen exhibited strong repellent activity ( 60 and $48 \%$, respectively) against $L$. serricorne at $4 \mathrm{~h}$ after exposure. In general, the variety of the repellent activity was easily affected by the sensitivity of the insects, the testing concentration, and the exposure duration. The repellent effect is complicated to interpret, and thus further work is needed to clarify it.

The results of the repellent assays showed that the EO and nine individual compounds exhibited different levels of repellent activity against $T$. castaneum at the highest testing concentration when exposed for $2 \mathrm{~h}$. The PR values of the EO, limonene, $\beta$-caryophyllene, $\rho$-cymen, $\alpha$-terpineol, 1,8 -cineole, acetophenone, and $\rho$-cymen-7-ol were all almost $100 \%$, and no statistically significant differences occurred between them. Although limonene accounted for $22 \%$ of the total EO, it exhibited weaker repellent activity than the EO against T. castaneum at all testing concentrations, while the EO was still highly repellent against T. castaneum. In this situation, the repellent activity of the EO did not seem to be greatly affected by limonene, and it is speculated that secondary components of the EOs may play an important role in the repellent activity, and have a synergistic effect that enhances the active effect. Another speculation is that some relatively inactive compounds play an important role in the multicomponent mixture. Some compounds are not significantly active on their own, but can enhance the active effects of other compounds. The repellent activity of the main components in EOs does not completely determine the overall effect of EOs, but synergistic and antagonistic interactions between the various components may have an important effect on the overall activity. Trongtokit et al. [53] reported that interactions between two or more components are caused by different ratios. The appropriate combination of compounds can increase the synergistic effect considerably. Compound proportioning is a complex and interesting issue that deserves further investigation. 

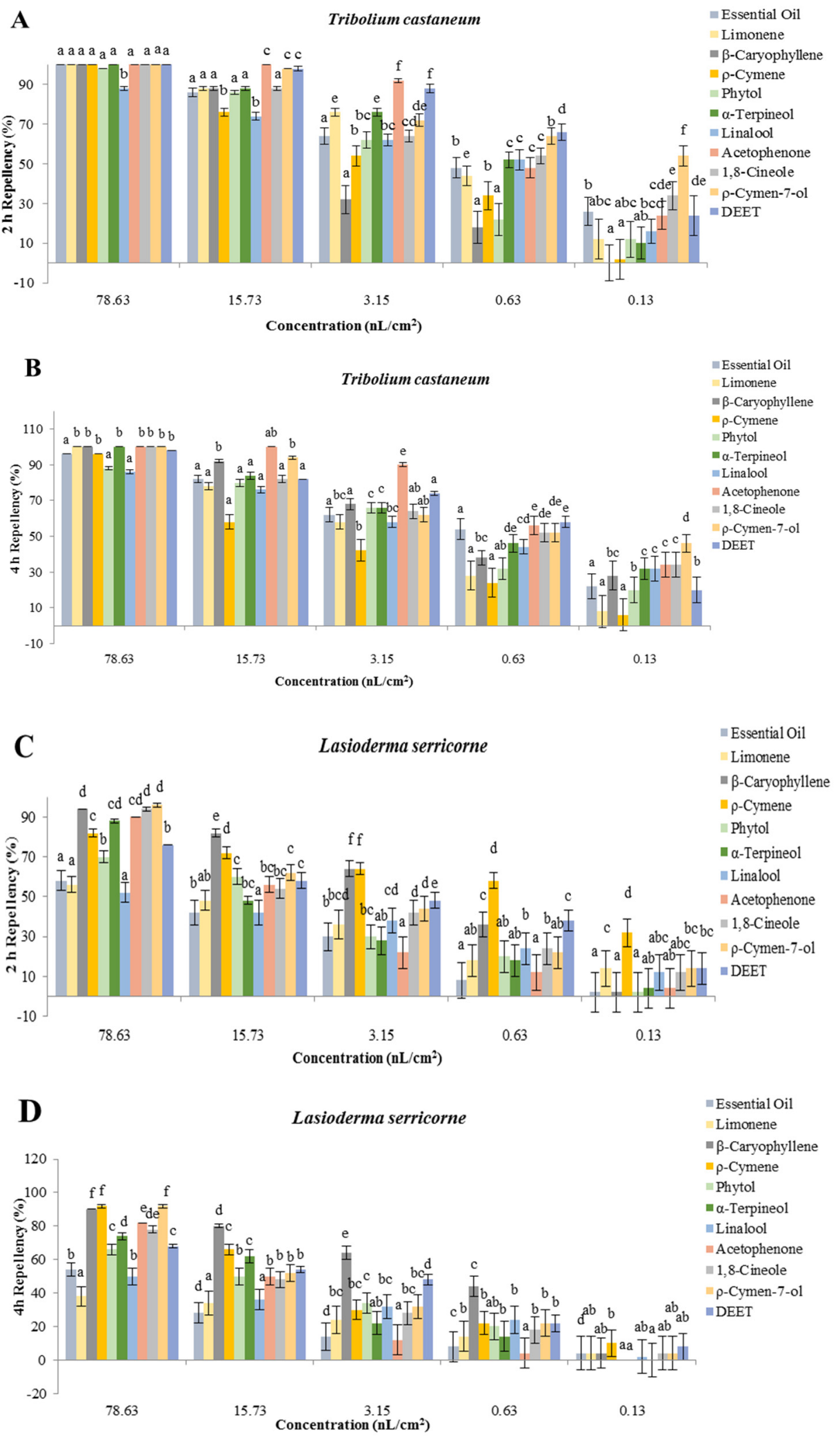

Figure 2. Percentage repellency (PR) of EO and compounds extracted from the E. densa against T. castaneum and L. serricorne at $2 \mathrm{~h}(\mathbf{A}, \mathbf{C})$ and $4 \mathrm{~h}(\mathbf{B}, \mathbf{D})$ after exposure. Differences between PR values of EO and DEET at the same concentration were determined by $t$-test $(p<0.05)$. At the same concentration, means ( \pm standard deviation) noted by the same lowercase letter do not differ significantly. 


\section{Conclusions}

In this work, the EO extracted from E. densa and its chemical compounds exhibited effective insecticidal toxicity and repellency against T. castaneum and L. serricorne. Compared with synthetic chemical insecticides, plant-derived materials have many advantages in pest management. They are scalable, safe for human health, and environmentally friendly. E. densa is used in traditional Chinese medicine, and has a long history. The characteristics highlighted above increase the feasibility of developing spice plants from the Elsholtzia family into eco-friendly pesticides, and suggest that the EO of Elsholtzia may have promise as a bioinsecticide or green repellent for pest management in warehouses and grain stores. However, further work should be devoted to investigating the mutual interactions among individual compounds, including their mechanism of action and possible relationships between toxicity and repellency. Meanwhile, further work should also be devoted to the effect of temperature on insecticidal and repellant activity. Whether the EO is actually safe for humans and the environment in the long term requires further experiments to verify. In addition, the target of action against insects should be confirmed.

Author Contributions: Conceptualization, J.L., J.W. and Y.S.; Funding acquisition, J.Z. and J.W.; Investigation, J.L., Y.S., H.W., Y.A. and W.K.; Validation, J.L., Y.S. and H.W.; Writing-original draft preparation, J.L. and Y.S.; Writing—review and editing, J.L., Y.S. and H.W.; Supervision, J.W. and J.Z.; Project administration, J.L. All authors have read and agreed to the published version of the manuscript.

Funding: This project was supported by the National Natural Science Foundation of China (No. 51873175) and Industry Pillar Program of Gansu Province (2020C-21).

Institutional Review Board Statement: Not applicable.

Conflicts of Interest: The authors declare no conflict of interest.

\section{References}

1. Hubert, J.; Stejskal, V.; Athanassiou, C.G.; Throne, J.E. Health hazards associated with arthropod infestation of stored products. Annu. Rev. Entomol. 2018, 63, 553-573. [CrossRef] [PubMed]

2. Thomas, W.P.; Throne, J.E. Biorational approaches to managing stored-product insects. Annu. Rev. Entomol. 2010, 55, 375-397. [CrossRef]

3. Boyer, S.; Zhang, H.; Lempérière, G. A review of control methods and resistance mechanisms in stored-product insects. Bull. Entomol. Res. 2012, 102, 213-229. [CrossRef] [PubMed]

4. Suman, C.; Rupinder, K.K.; Alka, S.; David, M.C.; Colin, J.B.; Rakesh, S.; Jagat, R.K. Progress on Azadirachta indica based biopesticidesin replacing synthetic toxic pesticides. Front. Plant Sci. 2017, 8, 610. [CrossRef]

5. Yadav, I.C.; Devi, N.L.; Syed, J.H.; Cheng, Z.; Li, J.; Zhang, G.; Jones, K.C. Current status of persistent organic pesticides residues in air, water, and soil, and their possible effect on neighboring countries: A comprehensive review of India. Sci. Total Environ. 2015, 511, 123-137. [CrossRef]

6. Baskar, K.; Ignacimuthu, S. Antifeedant, larvicidal and growth inhibitory effects of ononitol monohydrate isolated from Cassia tora L. against Helicoverpa armigera (Hub.) and Spodoptera litura (Fab.) (Lepidoptera: Noctuidae). Chemosphere 2012, 88, $384-388$. [CrossRef]

7. Arthur, F.H. Grain protectants: Current status and prospects for the future. J. Stored Prod. Res. 1996, 32, 293-302. [CrossRef]

8. Duraipandiyan, V.; Ignacimuthu, S.; Paulraj, M.G. Antifeedant and larvicidal activities of Rhein isolated from the flowers of Cassia fistula L. Saudi J. Biol. Sci. 2011, 18, 129-133. [CrossRef]

9. Isman, M.B. Botanical insecticides, deterrents, and repellents in modern agriculture and an increasingly regulated world. Annu. Rev. Entomol. 2006, 51, 45-66. [CrossRef]

10. Varma, J.; Dubey, N.K. Efficacy of essential oils of Caesulia axillaris and Mentha arvensis against some storage pests causing biodeterioration of food commodities. Int. J. Food. Microbiol. 2001, 68, 207-210. [CrossRef]

11. Miresmailli, S.; Isman, M.B. Botanical insecticides inspired by plant-herbivore chemical interactions. Trends Plant Sci. 2014, 19, 29-35. [CrossRef]

12. Isman, M.B.; Grieneisen, M.L. Botanical insecticide research: Many publications, limited useful data. Trends Plant Sci. 2014, 19, 140-145. [CrossRef] [PubMed]

13. Pavela, R.; Benelli, G. Essential oils as ecofriendly biopesticides? Challenges and constraints. Trends Plant Sci. 2016, 21, 1000-1007. [CrossRef] [PubMed]

14. Xue, Y. Common types and advantages and disadvantages of plant-derived pesticides. Mod. Gard. 2007, 6, 22-23. [CrossRef] 
15. Wang, Y.; Zhang, L.T.; Feng, Y.X.; Zhang, D.; Du, S.S. Comparative evaluation of the chemical composition and bioactivities of essential oils from four spice plants (Lauraceae) against stored-product insects. Ind. Crops Prod. 2019, 140, 111640. [CrossRef]

16. Zhang, Z.; Guo, S.S.; Zhang, W.J.; Geng, Z.F.; Liang, J.Y.; Du, S.S.; Wang, C.F.; Deng, Z.W. Essential oil and polyacetylenes from Artemisia ordosica and their bioactivities against Tribolium castaneum Herbst (Coleoptera: Tenebrionidae). Ind. Crops Prod. 2017, 100, 132-137. [CrossRef]

17. Feng, Y.X.; Wang, Y.; Geng, Z.F.; Zhang, D.; Almaz, B.; Du, S.S. Contact toxicity and repellent efficacy of Valerianaceae spp. to three stored-product insects and synergistic interactions between two major compounds camphene and bornyl acetate. Ecotoxicol. Environ. Saf. 2020, 190, 106-110. [CrossRef]

18. You, C.X.; Guo, S.S.; Zhang, W.J.; Geng, Z.F.; Liang, J.Y.; Ning, L.; Du, S.S.; Deng, Z.W. Chemical Constituents of Murraya tetramera Huang and Their Repellent Activity against Tribolium castaneum. Molecules 2017, 22, 1379. [CrossRef]

19. Chen, Z.Y.; Guo, S.S.; Cao, J.Q.; Pang, X.; Geng, Z.F.; Wang, Y.; Zhang, Z.; Du, S.S. Insecticidal and repellent activity of essential oil from Amomum villosum Lour., its main compounds against two stored-product insects. Int. J. Food Prop. 2018, 21, $2265-2275$. [CrossRef]

20. Feng, Y.X.; Wang, Y.; Chen, Z.Y.; Guo, S.S.; Du, S.S. Efficacy of bornyl acetate and camphene from Valeriana officinalis essential oil against two storage insects. Environ. Sci. Poll. Res. 2019, 26, 16157-16165. [CrossRef]

21. Fu, G.L. Chinese Higher Plants; Qingdao Publishing House: Qingdao, China, 2004; pp. 544-554.

22. Ren, Q.R.; Wang, Y.N.; Wang, Y.; Chen, H.; Xin, W.Y.; Ma, D.W. In vitro antioxidant activity and anti-tumor activity of total flavonoids from Elshltzia densa Benth. Nat. Prod. Res. Dev. 2017, 29, 14-21. [CrossRef]

23. Shao, Y.Z.; Hou, Q.Z.; Xie, Z.Y.; Yang, Y.Y.; He, C.Y.; Zhou, F.; Zhang, J.; Liang, J.Y. Bioactivities and chemical constituents of essential oils extracted from Caryopteris mongholica Bunge against two stored product insects. J. Essen. Oil Bear. Plants 2021, 24, 22-30. [CrossRef]

24. Mukazayire, M.J.; Tomani, J.C.; Chalchat, J.C.; Stévigny, C.; Duez, P. Chemical composition, antimicrobial and antioxidant activities of the essential oil of Guizotia scabra and Microglossa pyrifolia from Rwanda. Planta Med. 2009, 75, 9. [CrossRef]

25. Liu, Z.L.; Ho, S.H. Bioactivity of the essential oil extracted from Evodia rutaecarpa Hook f. et Thomas against the grain storage insects, Sitophilus zeamais Motsch., Tribolium castaneum (Herbst). J. Stored Prod. Res. 1999, 35, 317-328. [CrossRef]

26. Zhang, J.S.; Zhao, N.N.; Liu, Q.Z. Repellent constituents of essential oil of Cymbopogon distans aerial parts against two storedproduct insects. J. Agric. Food Chem. 2011, 59, 9910-9915. [CrossRef]

27. Liang, J.Y.; Guo, S.S.; You, C.X.; Zhang, W.L.; Wang, C.F.; Geng, Z.F.; Deng, Z.W.; Du, S.S.; Zhang, J. Chemical constituents and insecticidal activities of Ajania fruticulosa essential oil. Chem. Biodiver. 2016, 13, 1053-1057. [CrossRef]

28. Sakuma, M. Probit analysis of preference data. Appl. Entomol. Zool. 1998, 33, 339-347. [CrossRef]

29. Liu, Y.; Si, J.Y.; Cao, L.; Jia, X.G.; Li, X.J. Chemical composition, Antimicrobial and antiviral activities of the essential oil of Elsholtzia densa Benth. Nat. Prod. Res. Dev. 2012, 24, 1070-1074. [CrossRef]

30. Talzi, V.P. A ${ }^{13} \mathrm{C}$ and ${ }^{1} \mathrm{H}$ NMR analysis of perfumes. Russ. J. Appl. Chem. 2006, 79, 107-116. [CrossRef]

31. Kazutoshi, S.; Jun-ichiro, H.; Mariko, K. Purification and structural analysis of volatile sesquiterpenes produced by Escherichia coli carrying unidentified terpene synthase genes from edible plants of the family Araliaceae. Biosci. Biotechnol. Biochem. 2018, 82, 978-985. [CrossRef]

32. Liu, J.H.; Chen, L.G. Isolation and identification of the main chemical components in perilla oil. Flavour Fragr. Cosmet. 1998, 1, 19-20.

33. Chu, S.S.; Feng, H.J.; Liu, Z.L. Composition of essential oil of Chinese Chenopodium ambrosioides and insecticidal activity against maize weevil, Sitophilus zeamais. Pest. Manag. Sci. 2011, 67, 714-718. [CrossRef]

34. Dirk, U.; Josef, Z.; Hans, B. Biosynthesis of the irregular monoterpene artemisia ketone, the sesquiterpene germacrene D and other isoprenoids in Tanacetum vulgare L. (Asteraceae). Phytochemistry 2004, 65, 2463-2470. [CrossRef]

35. Lou, H.X.; Li, X.; Zhu, Y.R. New monoterpene components in North China white fore. J. Pharm. Sci. 1992, 10, 752-757.

36. Guerrini, A.; Rossi, D.; Paganetto, G. Chemical characterization (GC/MS and NMR Fingerprinting) and bioactivities of SouthAfrican Pelargonium capitatum (L.) L' Her. (Geraniaceae) essential oil. Chem. Biodiver. 2011, 8, 624-642. [CrossRef]

37. Toshikazu, S.; Nobuaki, F.; Fumio, I. Structure and synthesis of a new monoterpenoidal carboxamide from the seeds of the Thai medicinal plant Acacia concinna. Chem. Pharm. Bull. 1997, 45, 148-151. [CrossRef]

38. Senthil, K.R.; Karthikeyan, K.; Paramasivan, T.P. An Efficient Procedure for the TEMPO-Catalyzed Oxidation of Alcohols to Aldehydes and Ketones Using Ferric Chloride Hexahydrate as Terminal Oxidant; NRC Research Press: Ottawa, ON, Canada, 2008; Volume 86, pp. 720-725. [CrossRef]

39. Xu, S.Q.; Dun, S.Q.; Yang, X.L.; Pan, Y. Isolation and determination of $\alpha$-pinene and 1, 8-cineole from Oleum Vitics Negundo. Mod. Chin. Med. 2008, 10, 11-18. [CrossRef]

40. Nanjing University of Science and Technology. A Method for the Synthesis of Primary Alcohols. China Patent 201710683922.0, 26 February 2019.

41. Dong, Z.Y.; Wang, H.; Ma, Y.X.; Lan, X.Z.; Chen, M. Chemical constituents from Herpetospermum caudigerum. Chin. Trad. Patent Med. 2019, 41, 341-344. [CrossRef]

42. Ren, B.R.; Xia, B.; Li, W.L.; Wu, J.L.; Zhang, H.Q. Chemical constituents of Stenoloma chusanum. Chin. Tradit. Herb. Drugs 2007, 38, 20-23. [CrossRef] 
43. Liang, J.Y.; You, C.X.; Guo, S.S.; Zhang, W.J.; Li, Y.; Geng, Z.F.; Wang, C.F.; Du, S.S.; Deng, Z.W.; Zhang, J. Chemical constituents of the essential oil extracted from Rhododendron thymifolium and their insecticidal activities against Liposcelis bostrychophila or Tribolium castaneum. Ind. Crops Prod. 2016, 79, 267-273. [CrossRef]

44. Jiang, C.H.; Liu, Q.Z.; Du, S.S.; Deng, Z.W.; Liu, Z.L. Essential oil composition and insecticidal activity of Evodia lepta (Spreng) Merr. root barks from China against two grain storage insects. J. Med. Plants Res. 2012, 6, 3464-3469. [CrossRef]

45. Chen, H.; Yang, K.; You, C.X. Chemical constituents and biological activities against Tribolium castaneum (Herbst) of the essential oil from Citrus wilsonii leave. J. Ser. Chem. Soc. 2014, 79, 1213-1222. [CrossRef]

46. Wu, Y.; Guo, S.S.; Huang, D.Y.; Wang, C.F.; Wei, J.Y.; Li, Z.H.; Sun, J.S.; Bai, J.F.; Tian, Z.F.; Wang, P.J.; et al. Contact and repellent activities of Zingober zerumbet (L.) Smith against Lasioderma serricorne. J. Oleo Sci. 2017, 66, 399-405. [CrossRef] [PubMed]

47. Pavela, R.; Sedlák, P. Post-application temperature as a factor influencing the insecticidal activity of essential oil from Thymus vulgaris. Ind. Crops Prod. 2018, 113, 46-49. [CrossRef]

48. Wang, Y.; You, C.X.; Yang, K. Bioactivity of essential oil of Zingiber pur pureum Rhizomes and its main compounds against two stored product insects. J. Econo. Entomol. 2015, 8, 925-932. [CrossRef] [PubMed]

49. Yang, K.; Wang, C.F.; You, C.X.; Geng, Z.F.; Sun, R.Q.; Guo, S.S.; Du, S.S.; Liu, Z.L.; Deng, Z.W. Bioactivity of essential oil of Litsea cubeba from China and its main compounds against two stroed product insects. J. Asia Pac. Entomol. 2014, 17, 459-466. [CrossRef]

50. Wu, Y.; Zhang, W.J.; Huang, D.Y.; Wang, Y.; Wei, J.Y.; Li, Z.H.; Sun, J.S.; Bai, J.F.; Tian, Z.F.; Wang, P.J.; et al. Chemical compositions and insecticidal activities of Alpinia kwangsiensis essential oil against Lasioderma serricorne. Molecules 2015, 20, 21939-21945. [CrossRef] [PubMed]

51. Wang, Y.; You, C.X.; Wang, C.F.; Yang, K.; Chen, R.; Zhang, W.J.; Du, S.S.; Geng, Z.F.; Deng, Z.W. Chemical constituents and insecticida activities of the essential oil from Amomum tsaoko against two stored-productinsects. J. Oleo Sci. 2014, 63, $1019-1026$. [CrossRef]

52. Guo, S.S.; You, C.X.; Liang, J.Y.; Zhang, W.J.; Yang, K.; Geng, Z.F.; Wang, C.F.; Du, S.S.; Lei, N. Essential oil of Amomum maximum Roxb, and its bioaxtivities against two stored-product insects. J. Oleo Sci. 2015, 64, 1307-1314. [CrossRef]

53. Trongtokit, Y.; Rongsriyam, Y.; Komalamisra, N.; Apiwathnasorn, C. Comparative repellency of 38 essential oils against mosquito bites. Phytother. Res. 2005, 19, 303-309. [CrossRef] 